\title{
Efeitos da Cirurgia Bariátrica no Consumo de Doces e Alimentos Gordurosos por Pacientes em seu Período Pré e Pós-Operatório
}

\author{
Effects of Bariatric Surgery on the Consumption of Sweets and Fatty Foods by Patients in their \\ Pre and Postoperative Period
}

\author{
Lineker Fernandes Dias ${ }^{1}$ \\ Orcid: https://orcid.org/0000-0002-6256-7139
}

Giovanna Lyssa de Castro Andrade ${ }^{3}$

Orcid: https://orcid.org/0000-0002-4110-7619

João Victor Silveira Machado de Campos 5

Orcid: https://orcid.org/0000-0002-8375-5526

\author{
Ana Clara Toledo Detoni ${ }^{2}$ \\ Orcid: https://orcid.org/0000-0002-7765-0824 \\ Gustavo Vilela Alves ${ }^{4}$ \\ Orcid: https://orcid.org/0000-0003-4890-9427 \\ Jordana Martins Machado Araújo ${ }^{6}$ \\ Orcid: https://orcid.org/0000-0002-7106-9334
}

\begin{abstract}
Resumo
INTRODUÇÃO: Pacientes submetidos à cirurgia bariátrica $(\mathrm{CB})$ relatam alterações em seus comportamentos alimentares que podem advir de causas comportamentais, anatômicas e neuroendócrinas decorrentes dos impactos da cirurgia. Entretanto, não há consenso na literatura científica sobre os impactos da CB nos comportamentos alimentares associados, especificamente, ao consumo de alimentos muito calóricos. OBJETIVO: Analisar a produção científica que discorra acerca da associação entre consumo de alimentos com alta densidade energética por pacientes e seus respectivos períodos de pré ou pós operatório da CB. MATERIAIS E MÉTODOS: Revisão integrativa de literatura realizada nas bases de dados SciELO, Medline e Lilacs. A coleta de dados ocorreu entre março a junho de 2020. Foram considerados, para análise deste estudo, artigos publicados após 2015 com propostas de investigação consonantes com o objetivo desta pesquisa. Foram excluídos da análise artigos duplicados entre as bases de dados, dissertações, teses, monografias, resumos acadêmicos e literatura cinza. RESULTADOS: A amostra inicial de artigos com aplicação dos critérios de inclusão e exclusão foi de 114 artigos. Após seleção buscando manuscritos que atendessem ao escopo dessa revisão, o número total de trabalhos selecionados para estudo do seu conteúdo foi de 33 artigos. CONCLUSÕES: Os artigos indicam que a alteração de consumo de doces e alimentos gordurosos em período pré-operatório decorre de alterações comportamentais visando perda de peso. Entretanto, após a $\mathrm{CB}$, a redução do consumo desses alimentos com alta densidade energética pode estar associada a alterações neuroendócrinas intestinais relacionadas à saciedade, bem como redução na palatabilidade desses grupos alimentares.

Palavras-chave: avaliação nutricional; manejo da obesidade; comportamento alimentar.
\end{abstract}

\section{Abstract}

INTRODUCTION: Patients undergoing bariatric surgery $(\mathrm{CB})$ report changes in their eating behaviors that may arise from behavioral, anatomical, and neuroendocrine causes resulting from the impact of the surgery. However, there is no consensus in the scientific literature on the impacts of $\mathrm{BC}$ on eating behaviors specifically associated with the consumption of high-

\footnotetext{
${ }^{1}$ Universidade Federal de Uberlândia. E-mail: lineker-fernandes@hotmail.com

${ }^{2}$ Instituto Master de Ensino Presidente Antônio Carlos - (IMEPAC) Araguari. E-mail: ana.clara.detoni@hotmail.com

${ }^{3}$ Instituto Master de Ensino Presidente Antônio Carlos (IMEPAC) Araguari. E-mail: giovannaandrad@hotmail.com

${ }^{4}$ Instituto Master de Ensino Presidente Antônio Carlos (IMEPAC) Araguari. E-mail: gustavovilelaalves@hotmail.com

${ }^{5}$ Graduando de Medicina do Centro Universitário IMEPAC - Araguari. E-mail: joaovictor.prev@hotmail.com

${ }^{6}$ Instituto Master de Ensino Presidente Antônio Carlos (IMEPAC) Araguari. E-mail: jordanamma@hotmail.com
} 
calorie foods. OBJECTIVE: To analyze the scientific production that discusses the association between consumption of foods with high energy density by patients and their respective periods of pre or postoperative BC. MATERIALS AND METHODS: Integrative literature review performed in SciELO, Medline, and Lilacs databases. Data collection took place between March and June 2020. For the analysis of this study, articles published after 2015 with research proposals in line with the objective of this research were considered. Duplicate articles between the databases, dissertations, theses, monographs, academic abstracts, and gray literature were excluded from the analysis. RESULTS: The initial sample of articles with the application of the inclusion and exclusion criteria were 114 articles. After selecting manuscripts that met the scope of this review, the total number of works selected for the study of its content was 33 articles. CONCLUSIONS: The articles indicate that the change in consumption of sweets and fatty foods in the preoperative period is due to behavioral changes aimed at weight loss. However, after BC, the reduction in the consumption of these foods with high energy density may be associated with intestinal neuroendocrine alterations related to satiety, as well as a reduction in the palatability of these food groups.

Keywords: nutritional assessment; obesity management; feeding behaviour.

\section{Introdução}

A obesidade é classificada como uma doença decorrente do acúmulo excessivo de gordura corporal ${ }^{1}$. É causada, principalmente, por uma ingestão alimentar maior do que a necessidade energética real do indivíduo ${ }^{2}$. Além disso, a obesidade é fator de risco para desenvolvimento de outras doenças crônicas não-transmissíveis ${ }^{2}$.

A cirurgia bariátrica $(\mathrm{CB})$ é uma das opções disponíveis para tratamento da obesidade. Entretanto, apesar das estratégias atuais para tratamento da doença, existe a possibilidade da sua recidiva com o passar dos anos $^{3}$. Uma justificativa para isso seria a inadequação dos hábitos alimentares dos pacientes após o procedimento cirúrgico ${ }^{4}$.

Pesquisadores relatam que parte dos indivíduos portadores de obesidade, candidatos à $\mathrm{CB}$ possuem dificuldades para alterar seus hábitos alimentares ${ }^{5}$. Alguns pacientes, no período pós-operatório da $\mathrm{CB}$, consomem leite, gorduras e doces em quantidades inadequadas. O leite é ingerido em valores abaixo do recomendado ${ }^{6}$. Já os alimentos ricos em gorduras $^{78}$, doces ${ }^{9} \mathrm{e}$ carboidratos $^{7}$ são consumidos em quantidades acima do recomendado.

$\mathrm{O}$ consumo excessivo de doces e gorduras por pacientes após a $\mathrm{CB}$ possui causas multifatoriais ${ }^{10}$. Alguns pesquisadores afirmam que o elevado consumo de doces pode ser proveniente de hábitos alimentares anteriores à cirurgia. Outros, que, após a CB, não ocorre entre os pacientes alteração na palatabilidade de doces que justifique $o$ aumento ou redução do seu consumo ${ }^{11} 12$. Já, o elevado consumo de gorduras entre esses indivíduos poderia ser decorrente da realização de refeições de pequeno volume após a CB. O consumo de gorduras, por outro lado, tem sido associado à menor sensação de saciedade entre os pacientes ${ }^{13}$.

Apesar da existência de pesquisas sobre os hábitos alimentar dos pacientes após a $\mathrm{CB}$, a ingestão de grupos alimentares específicos, como doces e alimentos gordurosos, por esses indivíduos, precisa ser melhor estudada. Isso, com vista a promover o melhor entendimento das consequências da CB na palatabilidade, regulação neuro-hormonal de saciedade e compulsão alimentar por alimentos mais calóricos ${ }^{14}$. Nesse sentido, a condução de mais investigações nessa área pode auxiliar na proposição de orientações dietéticas adequadas para esses pacientes. Isso, visando à perda e manutenção do peso corporal antes e após a $\mathrm{CB}^{15}$.

Ante o exposto, a hipótese do presente estudo é que exista associação entre o padrão de consumo de doces e alimentos gordurosos por pacientes obesos e seus respectivos períodos de pré e pósoperatório da CB. Dessa forma, o objetivo deste estudo foi analisar a produção científica que discorra acerca da associação entre consumo de alimentos com alta densidade energética por pacientes e seus respectivos períodos de pré ou pós- 
operatório da cirurgia bariátrica.

\section{Materiais e Métodos}

Trata-se de um estudo que empregou metodologia de revisão integrativa de literatura buscando responder à seguinte questão norteadora: "existe associação entre consumo de alimentos com alta densidade energética por pacientes e seus respectivos períodos de pré ou pós-operatório da cirurgia bariátrica?".

Para resolução desse problema de pesquisa, as etapas propostas por Cooper ${ }^{16}$ para condução de revisões integrativas foram seguidas: I) estabelecimento de hipótese de investigação; II) construção do banco de dados; III) avaliação dos artigos; IV) análise e interpretação dos artigos; V) apresentação.

As bases de dados nas quais a busca foi realizada foram: a Scientific Electronic Library Online (SciELO), a Medical Literature Analysis and Retrieval System on-line (Medline) e a Literatura Latinoamericana em Ciências da Saúde (Lilacs). A coleta de dados ocorreu entre o mês de março a junho de 2020.

Os termos utilizados para realização desta revisão de literatura foram compreendidos por descritores disponibilizados na Biblioteca Virtual de Saúde, Descritores em Ciências da Saúde (BVS, 2020). Dentre esses, foram empregados: Comportamento Alimentar; Obesidade; Cirurgia Bariátrica; Período Pré-Operatório; Período Pós-Operatório; Ganho de Peso; Doces; Gorduras Insaturadas. Suas respectivas versões em inglês, foram: Feeding Behavior; Obesity; Bariatric Surgery; Preoperative Period; Postoperative Period; Weight Gain; Candy; Unsaturated Fats. Em complemento, Operadores Booleanos foram empregados para estabelecer interação entre os descritores na busca por artigos científicos realizada nas bases de dados.

Os critérios de inclusão neste estudo foram: artigos publicados em revistas revisadas por pares; estudos divulgados em português e inglês, entre 2015 e 2020, com metodologias de estudo do tipo pesquisa experimental e quase-experimental ou revisões sistemáticas com proposta de investigação consonante à pergunta-problema desta revisão integrativa. Já, os critérios de exclusão foram: artigos já selecionados em uma das base de dados e presente em outra, materiais compreendidos por resumos científicos, monografias, dissertações ou teses, bem como publicações caracterizadas como literatura cinza, segundo a definição desse termo colocada por Scott-Findlay e Estabrooks ${ }^{17}$.

A síntese do material coletado foi disposta em quadros com colunas que especificassem o autor do trabalho, objetivo, metodologia empregada, principais resultados e conclusões. Essa mesma etapa da pesquisa foi realizada por dois pesquisadores que, ao final, fizeram uma análise comparativa entre os quadros construídos por ambos.

Por fim, os artigos aptos para o estudo foram avaliados através da análise descritiva para revisões integrativas propostas por Broome $^{18}$. Em seguida, foi efetuada a categorização dos dados coletados em grupos distintos que respeitassem temas consonantes.

A construção das tabelas e figuras deste trabalho foi realizada por meio do software Microsoft Office Excel 2016.

Esta pesquisa dispensa aprovação de Comitês de Ética em Pesquisa (CEP) por utilizar informações disponibilizadas em bases de dados científicas de consulta pública $^{19}$.

\section{Resultados}

A busca nas três bases de dados, como primeira etapa do estudo, resultou em uma amostra inicial total de 102 artigos. Especificamente, a base de dados SciELO apresentou 15 artigos, a base de dados Lilacs 23 artigos e na base de dados MedLine, foram encontrados 64 artigos. Soma-se a esse número 12 artigos que também atendiam aos critérios de inclusão e foram identificados com base nas listas de referências desses primeiros artigos encontrados, totalizando, assim, 114 artigos para análise. 
Figura 1 - Fluxograma de condução da revisão integrativa nas bases de dados SciELO, Medline e Lilacs, 2020.

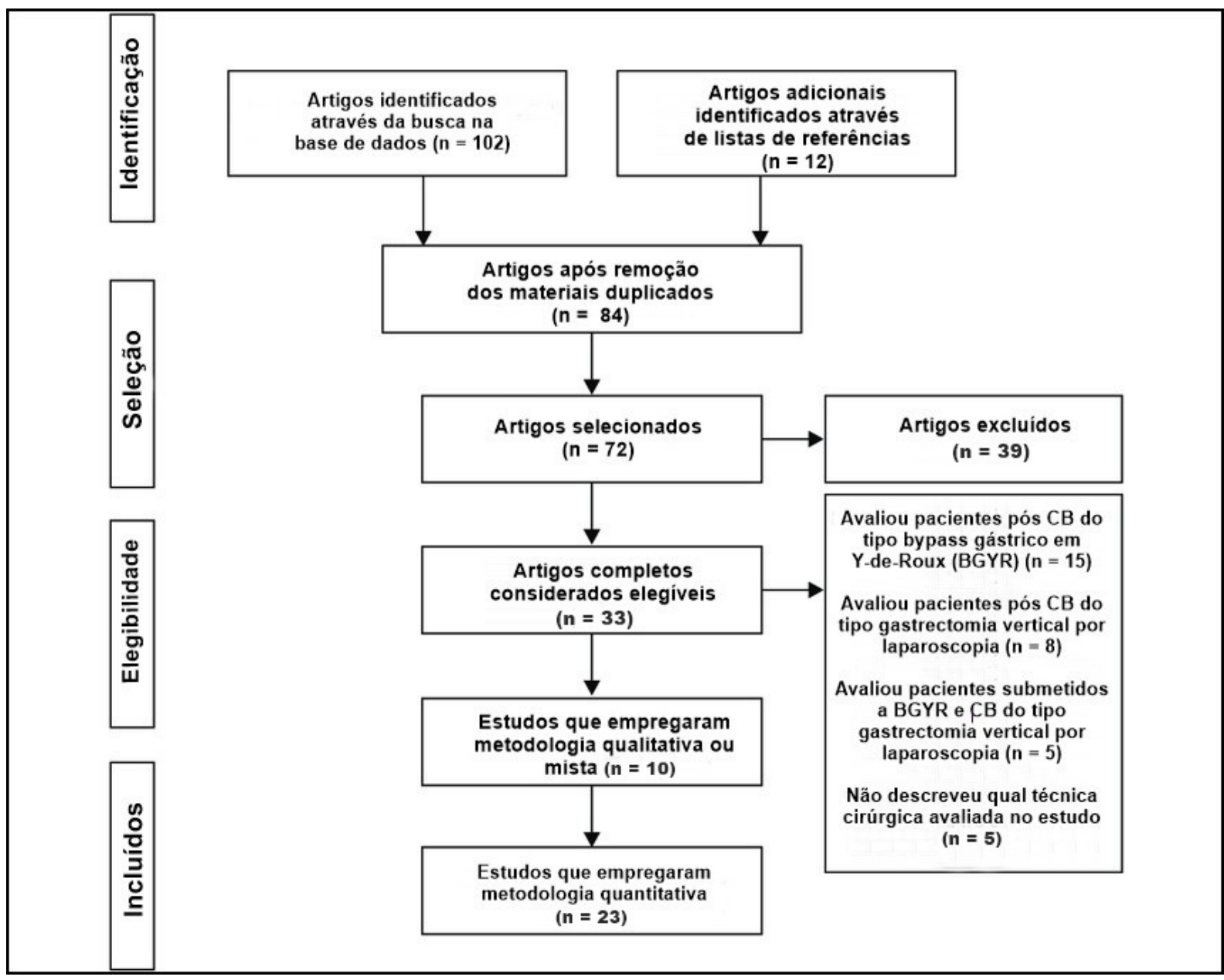

Fonte: Próprios autores, 2021.

Após remoção dos manuscritos duplicados entre as bases de dados, 84 artigos restaram para análise. Desse número, 72 artigos atendiam inicialmente ao escopo deste estudo. Seguidamente, com a aplicação final de todos os critérios de exclusão da primeira etapa, foram removidos 39 artigos da análise e a amostra final de artigos que satisfizeram os critérios deste estudo foi de 33 artigos em português e inglês. $\mathrm{O}$ fluxograma de seleção do material é trazido na Figura 1.

Quadro 1. Identificação dos artigos conforme título, autor (es), objetivo(s), principais resultados e seu ano de publicação, 2020.

\begin{tabular}{|c|c|c|c|c|c|}
\hline Artigo & Título & Autoria & Objetivo(s) & Resultados & Ano \\
\hline 1 & $\begin{array}{l}\text { Avaliação do } \\
\text { estado } \\
\text { nutricional de } \\
\text { pacientes pós- } \\
\text { operados } \\
\text { submetidos à } \\
\text { cirurgia } \\
\text { bariátrica. }\end{array}$ & $\begin{array}{c}\text { Leão, L. L.; } \\
\text { Abrantes, A. L. } \\
\text { O.; Gonçalves, R. } \\
\text { V.; De Souza, S. } \\
\text { A.; Soares, L. J. } \\
\text { F.; Farias, P. K. } \\
\text { S. }\end{array}$ & $\begin{array}{l}\text { "Avaliar os aspectos } \\
\text { nutricionais de } \\
\text { pacientes submetidos } \\
\text { à cirurgia bariátrica } \\
\text { em uma clínica } \\
\text { particular de Montes } \\
\text { Claros-MG" }\end{array}$ & $\begin{array}{l}75 \% \text { dos } \\
\text { pacientes após a CB } \\
\text { informaram consumir } \\
\text { leite do tipo desnatado. }\end{array}$ & 2018 \\
\hline
\end{tabular}


Efeitos da Cirurgia Bariátrica no Consumo de Alimentos Palatáveis Effects of Bariatric Surgery on the Consumption of Palatable Foods

\begin{tabular}{|c|c|c|c|c|c|}
\hline 2 & $\begin{array}{l}\text { Perfil Alimentar de } \\
\text { Pacientes Pós- } \\
\text { Cirurgia Bariátrica }\end{array}$ & $\begin{array}{c}\text { Paixão, A. L.; } \\
\text { Lourenço, V. V.; } \\
\text { Dias, J. S.; } \\
\text { Nogueira, A. A. } \\
\text { C. }\end{array}$ & $\begin{array}{c}\text { "Conhecer o perfil } \\
\text { alimentar de pacientes } \\
\text { pós cirurgia bariátrica } \\
\text { de uma clínica privada } \\
\text { de Belém-PA" }\end{array}$ & $\begin{array}{l}66.7 \% \text { dos } \\
\text { pacientes após a CB } \\
\text { relataram consumir } \\
\text { leite do tipo desnatado } \\
\text { diariamente. }\end{array}$ & 2018 \\
\hline 3 & $\begin{array}{l}\text { Reganho de peso } \\
\text { após cirurgia } \\
\text { bariátrica: avaliação } \\
\text { do perfil nutricional } \\
\text { dos pacientes } \\
\text { candidatos ao } \\
\text { procedimento de } \\
\text { plasma endoscópico } \\
\text { de argônio. }\end{array}$ & $\begin{array}{l}\text { Cambi, M. P. C.; } \\
\text { Marchesini, S. D. } \\
\text { e Baretta, G. A. } \\
\text { P. }\end{array}$ & $\begin{array}{c}\text { "Investigar nos } \\
\text { pacientes submetidos } \\
\text { à gastroplastia em Y- } \\
\text { de- Roux a recidiva de } \\
\text { peso, as deficiências } \\
\text { nutricionais }[. . .] "\end{array}$ & $\begin{array}{l}\text { Há consumo } \\
\text { elevado de doces em } \\
\text { valores acima do } \\
\text { recomendado entre } \\
\text { pacientes em período } \\
\text { pós-operatório da CB" }\end{array}$ & 2015 \\
\hline 4 & $\begin{array}{l}\text { Analysis of the } \\
\text { Association between } \\
\text { Eating Behaviors } \\
\text { and Weight Loss } \\
\text { after Laparoscopic } \\
\text { Sleeve Gastrectomy }\end{array}$ & $\begin{array}{l}\text { Nikiforova, I.; } \\
\text { Barnea, R.; } \\
\text { Azulai S. e } \\
\text { Susmallian, S. }\end{array}$ & $\begin{array}{l}\text { "Explorar a } \\
\text { influência de hábitos } \\
\text { alimentares anormais } \\
\text { no resultado da } \\
\text { gastrectomia vertical." }\end{array}$ & $\begin{array}{l}\text { Há consumo } \\
\text { de doces em valores } \\
\text { acima do recomendado } \\
\text { entre pacientes em } \\
\text { período pós-operatório } \\
\text { da CB }\end{array}$ & 2019 \\
\hline 5 & $\begin{array}{c}\text { Effect of } \\
\text { Preoperative Eating } \\
\text { Patterns and } \\
\text { Preoperative Weight } \\
\text { Loss on the Short- } \\
\text { and Mid-term } \\
\text { Weight Loss Results } \\
\text { of Sleeve } \\
\text { Gastrectomy }\end{array}$ & Ruiz-Tovar et al. & $\begin{array}{l}\text { “[...] avaliar o } \\
\text { efeito de diferentes } \\
\text { padrões alimentares } \\
\text { pré-operatórios e a } \\
\text { adesão a uma dieta } \\
\text { pré-operatória na } \\
\text { perda de peso pós- } \\
\text { operatória de curto e } \\
\text { médio prazo.” }\end{array}$ & $\begin{array}{l}\text { Há consumo } \\
\text { de doces em valores } \\
\text { acima do recomendado } \\
\text { entre pacientes em } \\
\text { período pós-operatório } \\
\text { da CB. }\end{array}$ & 2015 \\
\hline 6 & $\begin{array}{c}\text { Influence of } \\
\text { nutrition on } \\
\text { somatotropic axis: } \\
\text { Milk consumption in } \\
\text { adult individuals } \\
\text { with moderate- } \\
\text { severe obesity. }\end{array}$ & Barrea et al. & $\begin{array}{c}\text { “[...] avaliar o } \\
\text { consumo de leite por } \\
\text { meio do registro } \\
\text { alimentar de } 7 \text { dias em } \\
\text { indivíduos obesos". }\end{array}$ & $\begin{array}{l}\text { A maioria dos } \\
\text { pacientes candidatos à } \\
\text { CB relataram consumir } \\
\text { laticínios com baixo } \\
\text { teor de gordura. }\end{array}$ & 2015 \\
\hline 7 & $\begin{array}{l}\text { Dairy consumption } \\
\text { in association with } \\
\text { weight change and } \\
\text { risk of becoming } \\
\text { overweight or obese } \\
\text { in middle-aged and } \\
\text { older women: a } \\
\text { prospective cohort } \\
\text { study. }\end{array}$ & $\begin{array}{l}\text { Rautiainen, S.; } \\
\text { Wang, L.; Lee, I.- } \\
\text { M.; Manson, J. } \\
\text { E.; Buring, J. E.; } \\
\text { Sesso, H. D. }\end{array}$ & $\begin{array}{c}\text { “[...] investigar } \\
\text { prospectivamente } \\
\text { como a ingestão de } \\
\text { produtos lácteos } \\
\text { está associado à } \\
\text { mudança de peso e } \\
\text { risco de sobrepeso ou } \\
\text { obesidade em } \\
\text { mulheres" }\end{array}$ & $\begin{array}{l}\text { A maior parte } \\
\text { dos pacientes } \\
\text { candidatos à cirurgia } \\
\text { bariátrica avaliados } \\
\text { relataram consumir } \\
\text { laticínios com baixo } \\
\text { teor de gordura. }\end{array}$ & 2016 \\
\hline 8 & $\begin{array}{l}\text { Hedonic changes in } \\
\text { food choices } \\
\text { following Roux-en-Y } \\
\text { gastric bypass }\end{array}$ & $\begin{array}{l}\text { Hansen, T. T.; } \\
\text { Jakobsen, T. A.; } \\
\text { Nielsen, M. S.; } \\
\text { Sjödin, A.; Le } \\
\text { Roux, C. W.; } \\
\text { Schmidt, J. B. }\end{array}$ & $\begin{array}{c}\text { "resumir e discutir as } \\
\text { literatura científica } \\
\text { investigando } \\
\text { diferentes medidas } \\
\text { para avaliar } \\
\text { mudanças no impulso } \\
\text { para alimentos }\end{array}$ & $\begin{array}{l}\text { “Há tendência } \\
\text { dos pacientes em } \\
\text { reduzir o consumo de } \\
\text { alimentos muito } \\
\text { palatáveis e com alta } \\
\text { densidade energética }\end{array}$ & 2016 \\
\hline
\end{tabular}




\begin{tabular}{|c|c|c|c|c|c|}
\hline & & & $\begin{array}{c}\text { altamente palatáveis } \\
\text { após a CB" }\end{array}$ & $\begin{array}{c}\text { após a CB do tipo } \\
\text { BGYR “ }\end{array}$ & \\
\hline 9 & $\begin{array}{c}\text { Modificações } \\
\text { Comportamentais } \\
\text { entre o Pré e o Pós- } \\
\text { Operatório de } \\
\text { Pacientes } \\
\text { Bariátricos }\end{array}$ & $\begin{array}{l}\text { Justino, Y. A. C.; } \\
\text { Tatagiba, T. N. } \\
\text { B.; Pogian, L. P.; } \\
\text { Pimentel, F. C. }\end{array}$ & $\begin{array}{c}\text { "discutir a } \\
\text { função do alimentar-se } \\
\text { na vida do sujeito } \\
\text { antes da CB" }\end{array}$ & $\begin{array}{c}\text { Pacientes que } \\
\text { reforçaram em seu } \\
\text { relato de padrão de } \\
\text { consumo alimentar } \\
\text { características } \\
\text { prazerosas do sabor dos } \\
\text { alimentos } \\
\text { demonstraram maior } \\
\text { probabilidade de } \\
\text { consumi-los mais após } \\
\text { a CB. Exemplo: "Eu só } \\
\text { queria saber de comer } \\
\text { [...] lasanha, chocolate, } \\
\text { doce, pizza." }\end{array}$ & 2018 \\
\hline 10 & $\begin{array}{c}\text { Bariatric Surgery } \\
\text { Leads to Short-Term } \\
\text { Effects on Sweet } \\
\text { Taste Sensitivity and } \\
\text { Hedonic Evaluation } \\
\text { of Fatty Food } \\
\text { Stimuli } \\
\end{array}$ & $\begin{array}{l}\text { Nielsen, M. S.; } \\
\text { Andersen, I.; } \\
\text { Lange, B. et al. }\end{array}$ & $\begin{array}{c}\text { “[...] investigar o } \\
\text { efeito da cirurgia } \\
\text { bariátrica na } \\
\text { sensibilidade ao sabor } \\
\text { doce e na avaliação } \\
\text { hedônica de estímulos } \\
\text { alimentares doces, } \\
\text { salgados e } \\
\text { gordurosos" }\end{array}$ & $\begin{array}{c}\text { “O limiar de detecção } \\
\text { para o sabor doce } \\
\text { diminuiu após a perda } \\
\text { de peso induzida pela } \\
\text { dieta e } 6 \text { semanas após } \\
\text { o RYGB (ambos } \\
\text { P } \leq 0,03) . "\end{array}$ & 2019 \\
\hline 11 & $\begin{array}{c}\text { Taste and odor } \\
\text { preferences } \\
\text { following Roux-en-Y } \\
\text { surgery in humans }\end{array}$ & $\begin{array}{l}\text { Kittrell, H.; } \\
\text { Graber, W.; } \\
\text { Mariani, E.; } \\
\text { Czaja, K.; Hajnal, } \\
\text { A.; Di Lorenzo, } \\
\text { P. M. }\end{array}$ & $\begin{array}{c}\text { “[...] determinar a } \\
\text { relação entre } \\
\text { alterações nas } \\
\text { preferências de } \\
\text { paladar e odor e perda } \\
\text { de peso bem-sucedida } \\
\text { após cirurgia } \\
\text { bariátrica". }\end{array}$ & $\begin{array}{c}\text { “Os resultados } \\
\text { revelaram uma } \\
\text { mudança na preferência } \\
\text { por alimentos com } \\
\text { menos calorias." }\end{array}$ & 2018 \\
\hline 12 & $\begin{array}{l}\text { Predictors of lowest } \\
\text { weight and long- } \\
\text { term weight regain } \\
\text { among Roux-en-Y } \\
\text { gastric bypass } \\
\text { patients. }\end{array}$ & Yanos et al. & $\begin{array}{c}\text { “[...] avaliar a relação } \\
\text { de } \\
\text { bem documentado } \\
\text { (por exemplo, saúde, } \\
\text { dieta, atividade física) } \\
\text { após a CB” }\end{array}$ & $\begin{array}{l}\text { A não adesão a } \\
\text { orientações nutricionais } \\
\text { saudáveis está } \\
\text { associada ao reganho } \\
\text { de peso após CB. }\end{array}$ & 2015 \\
\hline 13 & $\begin{array}{c}\text { Associations } \\
\text { between Weight } \\
\text { Loss, Food Likes, } \\
\text { Dietary Behaviors, } \\
\text { and Chemosensory } \\
\text { Function in } \\
\text { Bariatric Surgery: A } \\
\text { Case-Control } \\
\text { Analysis in Women. }\end{array}$ & $\begin{array}{l}\text { Hubert, P. A.; } \\
\text { Papasavas, P.; } \\
\text { Stone, A. et al. }\end{array}$ & $\begin{array}{c}\text { “[...] comparar a } \\
\text { função } \\
\text { quimiossensorial, } \\
\text { gosto de alimentos e } \\
\text { comportamentos } \\
\text { alimentares em } \\
\text { mulheres com } \\
\text { obesidade mórbida } \\
\text { antes da cirurgia } \\
\text { bariátrica e aquelas em } \\
1 \text { ano após a cirurgia } \\
\text { bariátrica }[\ldots . . .\end{array}$ & $\begin{array}{l}\text { "Mostramos redução do } \\
\text { gosto de doçura na } \\
\text { sonda de doces e com o } \\
\text { gosto de doces e } \\
\text { bebidas doces (ou seja, } \\
\text { alimentos não } \\
\text { saudáveis), bem como } \\
\text { correlações } \\
\text { significativas entre o } \\
\text { gosto de doces de } \\
\text { amostra e o gosto de } \\
\text { doces (doces) e } \\
\text { picantes ou alimentos } \\
\text { saborosos (doces } \\
\text { picantes)." }\end{array}$ & 2019 \\
\hline
\end{tabular}




\begin{tabular}{|c|c|c|c|c|c|}
\hline 14 & $\begin{array}{c}\text { Alteration Pattern of } \\
\text { Taste Perception } \\
\text { After Bariatric } \\
\text { Surgery: A } \\
\text { Systematic Review } \\
\text { of Four Taste } \\
\text { Domains } \\
\end{array}$ & $\begin{array}{c}\text { Shoar, S.; } \\
\text { Naderan, M.; } \\
\text { Shoar, N.; } \\
\text { Modukuru, V.R.; } \\
\text { Mahmoodzadeh, } \\
\text { H. }\end{array}$ & $\begin{array}{l}\text { “[...] estudar o padrão } \\
\text { de alteração na } \\
\text { percepção de quatro } \\
\text { domínios gustativos } \\
\text { após diferentes } \\
\text { procedimentos } \\
\text { bariátricos”. }\end{array}$ & $\begin{array}{c}\text { “Nosso estudo mostrou } \\
\text { que a cirurgia bariátrica } \\
\text { está associada a uma } \\
\text { mudança significativa } \\
\text { na sensibilidade dos } \\
\text { quatro domínios do } \\
\text { paladar, especialmente } \\
\text { sabor de sal, doçura e } \\
\text { acidez.” }\end{array}$ & 2019 \\
\hline 15 & $\begin{array}{c}\text { Taste Changes after } \\
\text { Bariatric Surgery: a } \\
\text { Systematic Review. }\end{array}$ & $\begin{array}{c}\text { Ahmed, K.; } \\
\text { Penney, N.; } \\
\text { Darzi, A.; } \\
\text { Purkayastha, S. }\end{array}$ & $\begin{array}{c}\text { “[...] identificar todos } \\
\text { os artigos que } \\
\text { investigam gustação, } \\
\text { olfação e percepção } \\
\text { sensorial em estudos } \\
\text { em animais e } \\
\text { humanos, após } \\
\text { procedimentos } \\
\text { bariátricos." }\end{array}$ & $\begin{array}{l}\text { "A sensibilidade ao } \\
\text { sabor a estímulos doces } \\
\text { e gordurosos parece } \\
\text { aumentar no pós- } \\
\text { operatório. Além disso, } \\
\text { os pacientes também } \\
\text { têm uma resposta } \\
\text { hedônica reduzida a } \\
\text { esses estímulos." }\end{array}$ & 2018 \\
\hline 16 & $\begin{array}{l}\text { Food preferences } \\
\text { and underlying } \\
\text { mechanisms after } \\
\text { bariatric surgery. }\end{array}$ & $\begin{array}{l}\text { Behary, P.; } \\
\text { Miras, A.D. }\end{array}$ & $\begin{array}{c}\text { “[...] explorar as } \\
\text { mudanças nas } \\
\text { preferências } \\
\text { alimentares como um } \\
\text { novo mecanismo que } \\
\text { contribui para a perda } \\
\text { de peso, e também } \\
\text { focar nos processos } \\
\text { subjacentes que } \\
\text { modulam o } \\
\text { comportamento } \\
\text { alimentar após a } \\
\text { cirurgia bariátrica." }\end{array}$ & $\begin{array}{l}\text { "Pacientes após o } \\
\text { bypass gástrico têm } \\
\text { menos fome e preferem } \\
\text { opções alimentares } \\
\text { mais saudáveis. Eles } \\
\text { desenvolvem uma } \\
\text { acuidade aumentada ao } \\
\text { sabor doce, que é } \\
\text { percebido como mais } \\
\text { intenso. Os hormônios } \\
\text { intestinais podem ser os } \\
\text { mediadores dessas } \\
\text { alterações }[. . .] "\end{array}$ & 2015 \\
\hline 17 & $\begin{array}{c}\text { Long-Term } \\
\text { Modulation of } \\
\text { Appetitive } \\
\text { Hormones and } \\
\text { Sweet Cravings } \\
\text { After Adjustable } \\
\text { Gastric Banding and } \\
\text { Roux-en-Y Gastric } \\
\text { Bypass } \\
\end{array}$ & $\begin{array}{l}\text { Tsouristakis, } \\
\text { A.I.; Febres, G., } \\
\text { McMahon, D.J. } \\
\text { et al. } \\
\text {. }\end{array}$ & $\begin{array}{c}\text { “[...] quantificar as } \\
\text { alterações hormonais } \\
\text { que regulam a } \\
\text { homeostase energética } \\
\text { e as sensações } \\
\text { apetitivas antes e após } \\
\text { LAGB }(\mathrm{n}=18) \text { e } \\
\text { RYGB }(\mathrm{n}=38) \text {, a fim } \\
\text { de melhor } \\
\text { compreender os } \\
\text { mecanismos } \\
\text { subjacentes à maior } \\
\text { perda de peso após o } \\
\text { RYGB”. }\end{array}$ & $\begin{array}{c}\text { "Os desejos doces } \\
\text { diminuíram após a CB } \\
\text { do tipo BGYR." }\end{array}$ & 2019 \\
\hline 18 & $\begin{array}{l}\text { Shifts in Food } \\
\text { Preferences After } \\
\text { Bariatric Surgery: } \\
\text { Observational } \\
\text { Reports and } \\
\text { Proposed } \\
\text { Mechanisms. }\end{array}$ & $\begin{array}{l}\text { Kapoor, N.; Al- } \\
\text { Najim, W.; le } \\
\text { Roux, C.W.; } \\
\text { Docherty, N.G. }\end{array}$ & $\begin{array}{c}\text { “[...] revisar a } \\
\text { literatura disponível } \\
\text { que documenta as } \\
\text { mudanças na } \\
\text { preferência alimentar } \\
\text { em humanos e animais } \\
\text { experimentais após o } \\
\text { RYGB e discute a } \\
\text { teoria atual sobre os } \\
\text { mecanismos }\end{array}$ & $\begin{array}{l}\text { “A obesidade está } \\
\text { associada a uma } \\
\text { preferência crescente } \\
\text { por alimentos doces e } \\
\text { com alto teor de } \\
\text { gordura. Isso se altera } \\
\text { após a CB.” }\end{array}$ & 2017 \\
\hline
\end{tabular}




\begin{tabular}{|c|c|c|c|c|c|}
\hline & & & $\begin{array}{l}\text { subjacentes } \\
\text { envolvidos." }\end{array}$ & & \\
\hline 19 & $\begin{array}{c}\text { Changes in Dietary } \\
\text { Intake and Eating } \\
\text { Behavior in } \\
\text { Adolescents After } \\
\text { Bariatric Surgery: } \\
\text { an Ancillary Study } \\
\text { to the Teen-LABS } \\
\text { Consortium. }\end{array}$ & $\begin{array}{c}\text { Sarwer, D.B.; } \\
\text { Dilks, R.J.; } \\
\text { Spitzer, J.C. et al. }\end{array}$ & $\begin{array}{c}\text { “[...] investigar } \\
\text { mudanças no consumo } \\
\text { alimentar e no } \\
\text { comportamento } \\
\text { alimentar de } \\
\text { adolescentes com } \\
\text { obesidade submetidos } \\
\text { à cirurgia bariátrica ( } \mathrm{n} \\
\text { =119) ou modificação } \\
\text { do estilo de vida }(\mathrm{n}= \\
\text { 169).” }\end{array}$ & $\begin{array}{c}\text { “Após a cirurgia } \\
\text { bariátrica, maior perda } \\
\text { de peso entre os meses } \\
6 \text { e } 12 \text { do pós- } \\
\text { operatório foi associada } \\
\text { à consciência } \\
\text { autorreferida de desejo } \\
\text { por doces e consumo de } \\
\text { zinco." }\end{array}$ & 2017 \\
\hline 20 & $\begin{array}{l}\text { Altered neural } \\
\text { responsivity to food } \\
\text { cues in relation to } \\
\text { food preferences, } \\
\text { but not appetite- } \\
\text { related hormone } \\
\text { concentrations after } \\
\text { RYGB-surgery. }\end{array}$ & $\begin{array}{l}\text { Zoon, H.F.A; de } \\
\text { Bruijn, S.E.M; } \\
\text { Smeets, P.A.M. } \\
\text { et al. }\end{array}$ & $\begin{array}{c}\text { “[...] elucidar os } \\
\text { mecanismos } \\
\text { potenciais subjacentes } \\
\text { a essa mudança nas } \\
\text { preferências } \\
\text { alimentares, avaliando } \\
\text { as mudanças nas } \\
\text { respostas neurais às } \\
\text { imagens e odores dos } \\
\text { alimentos antes e } \\
\text { depois do RYGB." }\end{array}$ & $\begin{array}{c}\text { “Os pacientes } \\
\text { demonstraram uma } \\
\text { mudança nas } \\
\text { preferências } \\
\text { alimentares de produtos } \\
\text { com alto teor de } \\
\text { gordura / doce e } \\
\text { alimentos com baixo } \\
\text { consumo de energia / } \\
\text { salgados, que se } \\
\text { correlacionaram com a } \\
\text { diminuição da } \\
\text { responsividade superior } \\
\text { do lóbulo parietal ao } \\
\text { odor alimentar" }\end{array}$ & 2018 \\
\hline 21 & $\begin{array}{l}\text { Dopamine Links } \\
\text { Gastrointestinal } \\
\text { Rerouting to Altered } \\
\text { Sweet Appetite. }\end{array}$ & $\begin{array}{c}\text { Han, W.; Tellez, } \\
\text { L.A.; Niu, J. et } \\
\text { al. }\end{array}$ & $\begin{array}{c}\text { "[...] mostrar que uma } \\
\text { intervenção de bypass } \\
\text { duodenal-jejunal } \\
\text { (DJB) inibe o apetite } \\
\text { doce desadaptativo, } \\
\text { agindo em circuitos } \\
\text { estriatais responsivos } \\
\text { à dopamina" }\end{array}$ & $\begin{array}{l}\text { “O DJB interrompeu a } \\
\text { capacidade da } \\
\text { exposição recorrente ao } \\
\text { açúcar ao promover o } \\
\text { apetite doce em animais } \\
\text { saciados, revelando } \\
\text { assim uma ligação entre } \\
\text { o influxo de açúcar } \\
\text { duodenal recorrente e a } \\
\text { ingestão inadequada de } \\
\text { doce. Nossas } \\
\text { descobertas apontam } \\
\text { para um nexo de } \\
\text { causalidade entre a } \\
\text { sinalização da } \\
\text { dopamina estriatal e os } \\
\text { resultados de } \\
\text { intervenções } \\
\text { bariátricas.” }\end{array}$ & 2016 \\
\hline 22 & $\begin{array}{c}\text { Changes in taste } \\
\text { function and } \\
\text { ingestive behavior } \\
\text { following bariatric } \\
\text { surgery. }\end{array}$ & $\begin{array}{c}\text { Nance, K.; } \\
\text { Acevedo, M.B.; } \\
\text { Pepino, M.Y. }\end{array}$ & $\begin{array}{c}\text { “[...] resumir a } \\
\text { literatura sobre } \\
\text { mudanças na função } \\
\text { do paladar e no } \\
\text { comportamento } \\
\text { ingestivo após o CB } \\
\text { do tipo BGYR." }\end{array}$ & $\begin{array}{l}\text { “Descobrimos que } \\
\text { estudos usando } \\
\text { pesquisas e } \\
\text { questionários } \\
\text { geralmente descobrem } \\
\text { que os indivíduos } \\
\text { relatam alterações no } \\
\text { paladar e diminuem } \\
\text { suas preferências e }\end{array}$ & 2020 \\
\hline
\end{tabular}


Efeitos da Cirurgia Bariátrica no Consumo de Alimentos Palatáveis Effects of Bariatric Surgery on the Consumption of Palatable Foods

\begin{tabular}{|c|c|c|c|c|c|}
\hline & & & & $\begin{array}{l}\text { desejos por alimentos } \\
\text { com muita energia } \\
\text { (principalmente doces e } \\
\text { gordura)" }\end{array}$ & \\
\hline 23 & $\begin{array}{l}\text { Taste, Enjoyment, } \\
\text { and Desire of } \\
\text { Flavors Change } \\
\text { After Sleeve } \\
\text { Gastrectomy-Short } \\
\text { Term Results. }\end{array}$ & $\begin{array}{l}\text { Van Vuuren, } \\
\text { M.A.J; Strodl, E., } \\
\text { White, K.M., } \\
\text { Lockie, P.D. }\end{array}$ & $\begin{array}{c}\text { “[...] investigar a } \\
\text { satisfação com a } \\
\text { alimentação e a } \\
\text { mudança na percepção } \\
\text { do paladar, desejo e } \\
\text { apreciação das } \\
\text { alterações do sabor } \\
\text { pós CB" }\end{array}$ & $\begin{array}{c}\text { "A maioria dos } \\
\text { participantes relatou um } \\
\text { aumento na intensidade } \\
\text { do sabor doce ( } 60, \\
55 \%) \text { e associado à } \\
\text { alimentos gordurosos } \\
(57,70 \%) \text {. Os } \\
\text { participantes também } \\
\text { relataram uma } \\
\text { diminuição do prazer } \\
\text { pelos sabores doce ( } 77, \\
61 \%) "\end{array}$ & 2017 \\
\hline 24 & $\begin{array}{l}\text { The Effect of Roux- } \\
\text { en-Y Gastric Bypass } \\
\text { and Sleeve } \\
\text { Gastrectomy } \\
\text { Surgery on Dietary } \\
\text { Intake, Food } \\
\text { Preferences, and } \\
\text { Gastrointestinal } \\
\text { Symptoms in Post- } \\
\text { Surgical Morbidly } \\
\text { Obese Lebanese } \\
\text { Subjects: A Cross- } \\
\text { Sectional Pilot } \\
\text { Study. }\end{array}$ & $\begin{array}{l}\text { El Labban, S.; } \\
\text { Safadi, B.; Olabi, } \\
\text { A. }\end{array}$ & $\begin{array}{c}\text { “[...] comparar } \\
\text { retrospectivamente a } \\
\text { ingestão alimentar, as } \\
\text { preferências } \\
\text { alimentares e os } \\
\text { sintomas } \\
\text { gastrointestinais em } \\
\text { indivíduos com } \\
\text { obesidade extrema } \\
\text { após bypass gástrico } \\
\text { em Y de Roux } \\
\text { (RYGB) e } \\
\text { gastrectomia vertical } \\
\text { (SG).” }\end{array}$ & $\begin{array}{l}\text { “Não houve grandes } \\
\text { diferenças na ingestão } \\
\text { alimentar e preferências } \\
\text { alimentares entre os } \\
\text { grupos. Houve uma } \\
\text { tendência para comer } \\
\text { doce em indivíduos } \\
\text { com SG, com menos } \\
\text { sintomas de dumping, } \\
\text { sugerindo diferentes } \\
\text { mecanismos de ação } \\
\text { para cada } \\
\text { procedimento, o que } \\
\text { pode afetar o } \\
\text { comportamento } \\
\text { alimentar." }\end{array}$ & 2015 \\
\hline 25 & $\begin{array}{c}\text { The Relationship } \\
\text { Between Bariatric } \\
\text { Surgery and Diet } \\
\text { Quality: a } \\
\text { Systematic Review }\end{array}$ & $\begin{array}{l}\text { Zarshenas, N., } \\
\text { Tapsell, L.C., } \\
\text { Neale, E.P., } \\
\text { Batterham, M., e } \\
\text { Talbot, M. L. }\end{array}$ & $\begin{array}{c}\text { “[...] examinar a } \\
\text { relação entre cirurgia } \\
\text { bariátrica e qualidade } \\
\text { da dieta pelo menos } 1 \\
\text { ano após a cirurgia." }\end{array}$ & $\begin{array}{l}\text { Alimentos ricos em } \\
\text { gorduras são } \\
\text { consumidos em } \\
\text { quantidades acima do } \\
\text { recomendado após a } \\
\text { CB. }\end{array}$ & 2020 \\
\hline 26 & $\begin{array}{c}\text { Taste-related } \\
\text { reward is associated } \\
\text { with weight loss } \\
\text { following bariatric } \\
\text { surgery }\end{array}$ & $\begin{array}{l}\text { Smith, K.R.; } \\
\text { Papantoni, A.; } \\
\text { Veldhuizen, } \\
\text { M.G. et al. }\end{array}$ & $\begin{array}{c}\text { “[...] investigar } \\
\text { mudanças nas } \\
\text { preferências gustativas } \\
\text { e nas respostas } \\
\text { cerebrais induzidas } \\
\text { pelo paladar após o } \\
\text { bypass gástrico em Y } \\
\text { de Roux (RYGB) e } \\
\text { gastrectomia vertical } \\
\text { de manga (VSG) e } \\
\text { identificar potenciais } \\
\text { preditores } \\
\text { relacionados à perda } \\
\text { de peso relacionados } \\
\text { ao sabor.” }\end{array}$ & $\begin{array}{c}\text { “As alterações } \\
\text { anatômicas e / ou } \\
\text { metabólicas associadas } \\
\text { ao RYGB podem } \\
\text { "efetivamente redefinir" } \\
\text { o processamento neural } \\
\text { dos estímulos de } \\
\text { recompensa, resgatando } \\
\text { assim a ativação } \\
\text { contundente na via } \\
\text { mesolímbica } \\
\text { encontrada em } \\
\text { pacientes com } \\
\text { obesidade. Além disso, } \\
\text { esses achados sugerem }\end{array}$ & 2020 \\
\hline
\end{tabular}




\begin{tabular}{|c|c|c|c|c|c|}
\hline & & & & $\begin{array}{l}\text { que o RYGB pode ser } \\
\text { particularmente eficaz } \\
\text { em pacientes com } \\
\text { preferência por } \\
\text { alimentos doces." }\end{array}$ & \\
\hline 27 & $\begin{array}{l}\text { Effects of Sleeve } \\
\text { Gastrectomy vs. } \\
\text { Roux-en-Y Gastric } \\
\text { Bypass on Eating } \\
\text { Behavior and Sweet } \\
\text { Taste Perception in } \\
\text { Subjects with } \\
\text { Obesity. }\end{array}$ & $\begin{array}{c}\text { Nance, K.; } \\
\text { Eagon, J.C.; } \\
\text { Klein, S.; Pepino, } \\
\text { M.Y. }\end{array}$ & $\begin{array}{l}\text { "[...] testar a hipótese } \\
\text { de que a perda de peso } \\
\text { induzida pela CB do } \\
\text { tipo BGYR tem } \\
\text { maiores efeitos na } \\
\text { percepção do paladar e } \\
\text { no comportamento } \\
\text { alimentar do que a CB } \\
\text { do tipo gastrectomia } \\
\text { vertical }(\mathrm{GV}) . "\end{array}$ & $\begin{array}{l}\text { “Descobrimos que } \\
\text { ambos os tipos de } \\
\text { cirurgia não afetam a } \\
\text { sensibilidade à doçura } \\
\text { ou à salinidade. No } \\
\text { entanto, a perda de peso } \\
\text { induzida pela CB do } \\
\text { tipo BGYR ou GV } \\
\text { causaram a mesma } \\
\text { diminuição em: (1) } \\
\text { frequência de desejos } \\
\text { por alimentos; (2) } \\
\text { influência das emoções } \\
\text { e sinais alimentares } \\
\text { externos no } \\
\text { comportamento } \\
\text { alimentar; e (3) } \\
\text { mudança na } \\
\text { palatabilidade da } \\
\text { doçura passando de } \\
\text { agradável para } \\
\text { desagradável [...]” }\end{array}$ & 2017 \\
\hline 28 & $\begin{array}{c}\text { Link Between } \\
\text { Increased Satiety } \\
\text { Gut Hormones and } \\
\text { Reduced Food } \\
\text { Reward After } \\
\text { Gastric Bypass } \\
\text { Surgery for Obesity. }\end{array}$ & $\begin{array}{l}\text { Goldstone, A.P.; } \\
\text { Miras, A.D.; } \\
\text { Scholtz, S. et al. }\end{array}$ & $\begin{array}{c}\text { "[...] investigar o } \\
\text { papel da elevação dos } \\
\text { hormônios intestinais } \\
\text { de saciedade após a } \\
\text { CB do tipo BGYR, no } \\
\text { circuito de } \\
\text { recompensa } \\
\text { alimentar" }\end{array}$ & $\begin{array}{c}\text { “Respostas } \\
\text { aprimoradas dos } \\
\text { hormônios intestinais } \\
\text { de saciedade após CB } \\
\text { do tipo BGYR podem } \\
\text { ser um mecanismo } \\
\text { causal pelo qual } \\
\text { alterações anatômicas } \\
\text { do intestino em } \\
\text { cirurgias de obesidade } \\
\text { modificam as respostas } \\
\text { comportamentais e de } \\
\text { recompensa cerebral } \\
\text { aos alimentos.” }\end{array}$ & 2016 \\
\hline 29 & $\begin{array}{c}\text { Poor Health } \\
\text { Behaviors Prior to } \\
\text { Laparoscopic Sleeve } \\
\text { Gastrectomy } \\
\text { Surgery }\end{array}$ & $\begin{array}{l}\text { Oved, I.; Vaiman, } \\
\text { I.M.; Hod, K.; } \\
\text { Mardy-Tilbor, L.; } \\
\text { Torban, Y.; Sherf } \\
\text { Dagan, S. }\end{array}$ & $\begin{array}{c}\text { "[...] avaliar } \\
\text { comportamentos } \\
\text { alimentares e } \\
\text { tendências de estilo de } \\
\text { vida entre candidatos } \\
\text { à gastrectomia vertical } \\
\text { por laparoscopia } \\
\text { (LSG) e comparar } \\
\text { essas tendências entre } \\
\text { os sexos." }\end{array}$ & $\begin{array}{l}\text { “Alta ocorrência de } \\
\text { hábitos alimentares não } \\
\text { saudáveis e um estilo } \\
\text { de vida não ativo foram } \\
\text { detectados em obesos } \\
\text { mórbidos candidatos à } \\
\text { cirurgia de LSG." }\end{array}$ & 2017 \\
\hline
\end{tabular}


Efeitos da Cirurgia Bariátrica no Consumo de Alimentos Palatáveis Effects of Bariatric Surgery on the Consumption of Palatable Foods

\begin{tabular}{|c|c|c|c|c|c|}
\hline 30 & $\begin{array}{c}\text { Roux-En-Y Gastric } \\
\text { Bypass and Sleeve } \\
\text { Gastrectomy Does } \\
\text { Not Affect Food } \\
\text { Preferences When } \\
\text { Assessed by an Ad } \\
\text { libitum Buffet Meal. }\end{array}$ & $\begin{array}{l}\text { Nielsen, M.S.; } \\
\text { Christensen B.J.; } \\
\text { Ritz, C. et al. }\end{array}$ & $\begin{array}{c}\text { "[...] investigar se a } \\
\text { cirurgia com RYGB e } \\
\text { SG leva a mudanças } \\
\text { nas preferências } \\
\text { alimentares" }\end{array}$ & $\begin{array}{l}\text { “A redução na ingestão } \\
\text { de energia após a } \\
\text { cirurgia RYGB e SG e } \\
\text { a subsequente perda de } \\
\text { peso parecem estar } \\
\text { relacionadas } \\
\text { principalmente a uma } \\
\text { redução no tamanho } \\
\text { das porções e não por } \\
\text { alterações nas } \\
\text { preferências } \\
\text { alimentares em relação } \\
\text { a alimentos menos } \\
\text { densos em energia. } \\
\text { Estes resultados } \\
\text { sublinham a } \\
\text { necessidade de } \\
\text { investigar o } \\
\text { comportamento } \\
\text { alimentar visando o } \\
\text { comportamento direto." }\end{array}$ & 2017 \\
\hline 31 & $\begin{array}{l}\text { Food cravings and } \\
\text { food consumption } \\
\text { after Roux-en-Y } \\
\text { gastric bypass } \\
\text { versus } \\
\text { cholecystectomy. }\end{array}$ & $\begin{array}{l}\text { Sudan, R.; Sudan, } \\
\text { R.; Lyden, E., } \\
\text { Thompson, J.S. }\end{array}$ & $\begin{array}{c}\text { “[...] estudar os } \\
\text { desejos por alimentos } \\
\text { com alta densidade } \\
\text { energética em } \\
\text { pacientes após a CB } \\
\text { do tipo BGYR e } \\
\text { compará-los com os } \\
\text { pacientes com } \\
\text { controle de } \\
\text { colecistectomia (CC)" }\end{array}$ & $\begin{array}{l}\text { "Essas descobertas } \\
\text { indicam que a CB do } \\
\text { tipo BGYR pode } \\
\text { limitar o consumo de } \\
\text { alimentos, mas não } \\
\text { afeta o desejo de } \\
\text { consumir certos tipos } \\
\text { de alimentos. Como os } \\
\text { desejos alimentares são } \\
\text { altos em pacientes com } \\
\text { obesidade antes da } \\
\text { cirurgia e permanecem } \\
\text { altos após a cirurgia, } \\
\text { esses achados sugerem } \\
\text { uma possível razão para } \\
\text { o não cumprimento das } \\
\text { recomendações } \\
\text { alimentares após a CB.” }\end{array}$ & 2017 \\
\hline \multirow[t]{2}{*}{32} & $\begin{array}{l}\text { Food Intake and } \\
\text { Changes in Eating } \\
\text { Behavior After } \\
\text { Laparoscopic Sleeve } \\
\text { Gastrectomy }\end{array}$ & $\begin{array}{c}\text { Coluzzi, I.; } \\
\text { Raparelli, L.; } \\
\text { Guarnacci, L. et } \\
\text { al. }\end{array}$ & $\begin{array}{c}\text { “[...] avaliar as } \\
\text { alterações na ingestão } \\
\text { calórica e de } \\
\text { macronutrientes após } \\
\text { o LSG e a relação } \\
\text { entre alterações de } \\
\text { paladar e tolerância } \\
\text { alimentar ao longo de } \\
2 \text { anos.” }\end{array}$ & $\begin{array}{c}\text { "O LSG reduziu a } \\
\text { ingestão calórica, tanto } \\
\text { pelo volume de } \\
\text { alimentos quanto pela } \\
\text { densidade calórica dos } \\
\text { alimentos consumidos." }\end{array}$ & 2016 \\
\hline & $\begin{array}{l}\text { Effect of Roux-en-Y } \\
\text { gastric bypass and } \\
\text { sleeve gastrectomy } \\
\text { on taste acuity and }\end{array}$ & $\begin{array}{c}\text { El Labban, S.; } \\
\text { Safadi, B.; Olabi, } \\
\text { A. }\end{array}$ & $\begin{array}{l}\text { “[...] comparar } \\
\text { retrospectivamente a } \\
\text { acuidade gustativa e a } \\
\text { aceitabilidade da }\end{array}$ & $\begin{array}{l}\text { "O limiar de azedume } \\
\text { foi significativamente }\end{array}$ & \\
\hline
\end{tabular}




\begin{tabular}{|c|c|c|c|c|}
\hline 33 & $\begin{array}{c}\text { sweetness } \\
\text { acceptability in } \\
\text { postsurgical } \\
\text { subjects. }\end{array}$ & $\begin{array}{c}\text { doçura após o bypass } \\
\text { gástrico em Y de } \\
\text { Roux e gastrectomia } \\
\text { vertical." }\end{array}$ & $\begin{array}{c}\text { maior entre os } \\
\text { indivíduos que foram } \\
\text { submetidos à derivação } \\
\text { gástrica em Y de Roux } \\
(\mathrm{P}=0,0045) \text {. Nenhuma } \\
\text { outra diferença foi } \\
\text { obtida para os outros } \\
\text { limiares ou } \\
\text { aceitabilidade de } \\
\text { doçura" }\end{array}$ & $\begin{array}{c}2016 \\
\end{array}$ \\
\hline
\end{tabular}

A maioria dos artigos foi publicado em língua inglesa entre os anos de 2016 e 2017. Dos artigos selecionados para análise, 4 $(12.12 \%)$ deles são fruto de pesquisas conduzidas e veiculadas a periódicos do Brasil e $29(87.87 \%)$ deles de pesquisas publicadas em inglês. Acerca do ano de publicação, 6 (18.18\%) foram publicados em 2015, 7 (21.21\%) em 2016, 7 (20.58\%) em 2017, 5 (15.5\%) em 2018, 5 (15.5\%) em 2019 e 3 (9.09\%) em 2020.

Quando analisada a metodologia, a maior parte das pesquisas empregou investigação quantitativa sem descrever, precisamente, o teste estatístico utilizado. Numericamente, $23 \quad(69.69 \%)$ estudos empregaram em sua investigação uma metodologia quantitativa, $3 \quad(12.12 \%)$ qualitativa e $7(21.21 \%)$ mista. No que concerne à análise estatística realizada, 9 $(9.09 \%)$ artigos utilizaram o Teste de Qui Quadrado, 6 (18.18\%) a Correlação de Pearson, 2 (5.88\%) o teste ANOVA, 5 (15.5\%) o Teste Mann-Whitney e 10 (30.30\%) não citaram o teste estatístico empregado.

A maioria dos estudos avaliou o impacto da $\mathrm{CB}$ do tipo bypass gástrico em Yde-Roux (BGYR) no consumo alimentar dos pacientes. Nesse sentido, 15 (45.6\%) estudos avaliaram cirurgia do tipo BGYR, 8 (24.24\%) gastrectomia vertical por laparoscopia, 5 $(15.5 \%)$ ambas e $5(15.5 \%)$ não relataram qual o tipo de técnica cirúrgica avaliada.

\section{Discussão}

Inicialmente, os achados dessa revisão de literatura reforçaram a existência de inúmeras pesquisas na literatura científica que suportam a existência de uma associação entre consumo de alimentos com alta densidade energética por pacientes e seus respectivos períodos de pré ou pós-operatório da cirurgia bariátrica (CB). Especificamente, os resultados aqui encontrados, apontam que a $\mathrm{CB}$ do tipo bypass gástrico em Y de Roux (BGYR) tem sido a modalidade de $\mathrm{CB}$ mais associada a alterações de comportamentos alimentares. Comparando os achados desta revisão integrativa com demais trabalhos similares, constata-se que essa observação foi, também, verificada por outros pesquisadores ${ }^{35} 14$.

Os artigos analisados suportam que o comportamento alimentar mais alterado antes da $\mathrm{CB}$, entre os pacientes candidatos à cirurgia, foi reduzir o consumo de alimentos de alta densidade energética objetivando a perda de peso para cumprimento dos pré-requisitos para realização das diversas modalidades de CB. Outros pesquisadores relataram, também, aumento da adoção de hábitos alimentares saudáveis por pacientes em período préoperatório da $\mathrm{CB}^{32}$. Entretanto, esses hábitos são adotados motivando perda de peso, não por redução do desejo de consumo desses grupos alimentares. Em outras palavras, a adoção de novos hábitos alimentares não se justifica, nos estudos analisados, por alterações gustativas relatadas pelos pacientes ${ }^{32}$.

Outras pesquisas apontam que pacientes candidatos à $\mathrm{CB}$ relatam, também, consumir laticínios com baixo teor de gordura $^{25}{ }^{26}$. Esse padrão alimentar pode ocorrer em substituição ao consumo de bebidas de alta densidade energética ${ }^{25}{ }^{26}$. Nesse viés, é possível que esses pacientes relatam consumir laticínios desnatados ou semidesnatados por estarem em um período de 
alteração de seus hábitos alimentares objetivando perda de peso. Dessa forma, esse hábito alimentar, não necessariamente, advém de um período anterior ao pré-operatório da CB.

Diferentemente disso, após a $\mathrm{CB}$, a alteração de comportamento alimentar mais relatada pelos pacientes nos estudos analisados foi a redução do consumo de alimentos com alta densidade energética em associação à redução da sua palatabilidade. Há relatos na literatura científica de redução da percepção gustativa dos pacientes por alimentos muito palatáveis, como doces. Isso, especialmente, em períodos de acentuada perda de peso ou em pós-operatório recente ${ }^{49}$. Como exemplo, até 6 meses desde a realização da $\mathrm{CB}^{49}$. Nesse contexto, alguns estudos que utilizaram testes feitos com soluções de sacarose indicaram que, após a $\mathrm{CB}$, ocorreu redução na percepção da solução ao paladar dos pacientes. Faz-se importante observar que esse achado pode ser um mecanismo importante para impulsionar a perda de peso após a realização da cirurgia ${ }^{24}$. Nesse cenário, é sabido que a $\mathrm{CB}$ do tipo BGYR resulta em uma redução mais acentuada de impulsos alimentares entre os pacientes em seu período pós-operatório ${ }^{49}$.

Convém ressaltar, entretanto, que após a CB do tipo BGYR os pacientes são submetidos à dieta líquida por, minimamente, duas semanas. Dessa forma, o comportamento alimentar dos pacientes nesse período pósoperatório imediato deve ser avaliado criticamente $^{49}$.

Outro aspecto é que as preferências alimentares dos pacientes submetidos à $\mathrm{CB}$, por alimentos com alto teor de gordura, diminuíram após a cirurgia. Tal redução foi associada a uma resposta neural reduzida do lóbulo parietal desses indivíduos aos odores de alimentos gordurosos ${ }^{25}$. Além disso, há indicativos de que indivíduos submetidos à $\mathrm{CB}$ apresentem respostas mais aguçadas aos hormônios intestinais de saciedade após ingestão alimentar. Portanto, alterações neurohormonais de recompensa, após consumo alimentar, podem justificar a menor ingestão de alimentos obesogênicos no período pósoperatório $^{46}$.
Apesar disso, outros estudos indicam que a redução do consumo de alguns grupos alimentares está relacionada com uma diminuição do tamanho das porções alimentares após a CB. Isso, decorrente da diminuição do trato gastrointestinal que passa a não comportar mais grandes volumes de $\operatorname{alimentos}^{27}$ 49. Nesse sentido, tais pesquisadores advertem para cautela na interpretação das alterações gustativas relatadas por pacientes após a cirurgia, especialmente, as ligadas à $\mathrm{CB}$ do tipo BGYR.

\section{Conclusão}

Foram identificadas diferenças entre o consumo de alimentos com alta densidade energética por pacientes candidatos à $\mathrm{CB}$ e seus respectivos períodos de pré ou pósoperatório. Os artigos analisados indicaram que as alterações no padrão alimentar de pacientes em período pré-operatório da $\mathrm{CB}$ advêm, principalmente, da mudança de hábitos alimentares típicas desse período. Estas, objetivando perda de peso antes da cirurgia através de hábitos alimentares saudáveis. Já, as mudanças de padrão alimentar, sobretudo, entre pacientes em período pós-operatório da $\mathrm{CB}$, decorrem, em sua maioria, de alterações morfofisiológicas corporais secundárias à própria cirurgia. Estas, que alteram a regulação neuro-hormonal intestinal responsável pela saciedade, bem como a palatabilidade de alimentos obesogênicos. Dessa forma, resultando em perda do interesse desses pacientes por grupos alimentares como doces e gorduras.

Consideramos como limitação deste estudo o fato de não ter sido avaliada a qualidade metodológica dos trabalhos analisados. Ademais, comportamentos humanos possuem causas multifatoriais. Mais estudos fazem-se necessários para generalizações dos achados aqui colocados. Sugere-se, para tanto, que futuras revisões integrativas na área busquem avaliar estudos com aplicação de inquéritos alimentares diferentes dos empregados nos artigos aqui analisados, bem como pesquisas com acompanhamento 
longitudinal tardio dos pacientes após a CB. A melhoria de indicadores de saúde pública no Brasil associados à obesidade são resultados que podem advir da condução dessas investigações.

\section{Referências Bibliográficas}

1. World Health Organization. Obesity: Preventing and managing the global epidemic. Report of a WHO Consultation on Obesity. Geneva: WHO; 1998.

2. GUYTON AC, HALL JE. Tratado de Fisiologia Médica. $11^{\mathrm{a}}$ ed. Rio de Janeiro, Elsevier Ed., 2006.

3. Mechanick JI, Youdim A, Jones DB, Timothy GW, Hurley DL, Molly MM, et al. Clinical Practice Guidelines for the Perioperative Nutritional, Metabolic, and Nonsurgical Support of the Bariatric Surgery Patient—2013 Update: Cosponsored by American Association of Clinical Endocrinologists, The Obesity Society, and American Society for Metabolic \& Bariatric Surgery. Surgery for Obesity and Related Diseases. Weight Recidivism Post-Bariatric Surgery: A Systematic Review. Obesity Surgery, v. 23, n. 11, 2013. Disponível em: https://link.springer.com/article/10.1007\%2Fs11695-0131070-4. Acesso em: 20 Mar. 2020.

4. Fink-Miller E, Rigby A. The Utility of the Weight and Lifestyle Inventory (WALI) in Predicting 2-Year Weight Loss After Bariatric Surgery. Obesity Surgery, v. 27, n. 4, p. 933-939, 2016. Disponível em: https://link.springer.com/article/10.1007/s11695-0162385-8\#citeas. Acesso em: 20 Mar. 2020.

5. SOARES JM, MICHELETTI J, OLIVEIRA ML, SILVA ACG, CAVAGNARI MAV Food practices of patients in postoperative surgery of bariatric surgery: integration review. BRASPEN J, v. 32, n, 3, 2017. Disponível em: https://pesquisa.bvsalud.org/portal/resource/pt/biblio-906072. Acesso em: 20 Mar. 2020.

6. SOARES FL, BISSONI DE SOUSA L, CORRADI-PERINI C, RAMOS DA CRUZ MR, NUNES MGJ, et al. Food Quality in the Late Postoperative Period of Bariatric Surgery: An Evaluation Using the Bariatric Food Pyramid. Obesity Surgery, v. 24, n. 9, 2014. Disponível em: https://link.springer.com/article/10.1007/s11695-014-1198-x. Acesso em: 20 Mar. 2020.

7. ZARSHENAS N, TAPSELL LC, NEALE EP, BATTERHAM M, TALBOT ML. The Relationship Between Bariatric Surgery and Diet Quality: a Systematic Review. Obesity Surgery. 2020. Disponível em: https://pubmed.ncbi.nlm.nih.gov/31940138/. Acesso em: 20 Mar. 2020.

8. Coluzzi I, Raparelli L, Guarnacci L, Paone E, Del Genio G, le Roux CW, et al. Food Intake and Changes in Eating Behavior After Laparoscopic Sleeve Gastrectomy. Obesity Surgery, v. 26, n. 9, 2016. Disponível em:

https://link.springer.com/article/10.1007\%2Fs11695-015-2043-6. Acesso em: 20 Mar. 2020.

9. FREIRE RH, BORGES MC, ALVAREZ-LEITE JI, CORREIA MITD. Food quality, physical activity, and nutritional follow-up as determinant of weight regain after Rouxen-Y gastric bypass. Nutrition, v. 28, n. 1, 2012. Disponível em: https://www.sciencedirect.com/science/article/abs/pii/S0899900711000487?via\%3Dihub. Acesso em: 20 Mar. 2020.

10. Le Roux CW, Bueter M, Theis N, Werling M, Ashrafian H, Löwenstein C, et al. Gastric bypass reduces fat intake and preference. American Journal of Physiology-Regulatory, Integrative and Comparative Physiology. v. 301, n. 4, 2011. Disponível 
em:https://journals.physiology.org/doi/full/10.1152/ajpregu.00139.2011. Acesso em: 20 Mar. 2020.

11. MATHES CM, SPECTOR AC. Food selection and taste changes in humans after Rouxen-Y gastric bypass surgery: a direct-measures approach. Physiology \& behavior, v. 107, n. 4, p. 476-483, 2012. Disponível em:

https://www.sciencedirect.com/science/article/abs/pii/S0031938412000807?via\%3Dihub. Acesso em: 20 Mar. 2020.

12. Sugerman HJ, Kellum JM, Engle KM, Wolfe L, Starkey JV, Birkenhauer R, et al. Gastric bypass for treating severe obesity. Am J Clin Nutr, v. 55, n. 2, 1992. Disponível em: https://www.ncbi.nlm.nih.gov/pubmed/1733127. Acesso em: 20 Mar. 2020.

13. Kapoor N, Al-Najim W, le Roux CW, Docherty NG. Shifts in Food Preferences After Bariatric Surgery: Observational Reports and Proposed Mechanisms. Curr Obes Rep., v. 6, n. 3, p. 246-252, 2017. Disponível em: https://pubmed.ncbi.nlm.nih.gov/28699145/. Acesso em: 20 Mar. 2020.

14. Adler S, Fowler N, Robinson AH, Salcido L, Darcy A, Toyama H, et al. Correlates of Dietary Adherence and Maladaptive Eating Patterns Following Roux-en-Y Bariatric Surgery. Obesity Surgery, v. 28, n. 4, 2017. Disponível em: https://link.springer.com/article/10.1007\%2Fs11695-017-2987-9. Acesso em: 20 Mar. 2020.

15. NERY MA, CÂMERA VJ, SILVEIRA MS. REGANHO DE PESO PÓS CIRURGIA BARIÁTRICA. UMA REVISÃO DE LITERATURA. Revista Ciência (In) Cena, v. 1, n. 11, p. 40-52, 2020.

16. Cooper HM. The Integrative research review. Beverly Hills: SAGE Publications; 1984.

17. SCOTT-FINDLAY S, ESTABROOKS CA. Mapping the organizational culture research in nursing: a literature review. Journal of advanced nursing, v. 56, n. 5, p. 498-513, 2006.

18. Broome ME. Integrative literature reviews for the development of concepts. In: Rodgers BL, Knafl KA. Concept development in nursing: foundations, techniques, and applications. 2nd ed. Philadelphia: Saunders; c2000.

19. Brasil. Conselho Nacional de Saúde. Resolução no 510 , de 7 de abril de 2016. Diário Oficial da União, Poder Executivo, Brasília, DF, 24 maio 2016. Seção 1, n. 98, p. 44-6

20. LEÃO LL, ABRANTES ALO, GONÇALVES RV, SOUZA SA, SOARES LJF, FARIAS PKS. Avaliação do estado nutricional de pacientes pós-operados submetidos à cirurgia bariátrica. RBONE - Revista Brasileira de Obesidade, Nutrição e Emagrecimento, v. 12, n. 72, p. 439-445, 15 ago. 2018.

21. Paixão AL, Costa VVL, Dias JS, Nogueira AC. Perfil alimentar de pacientes pós cirurgia bariátrica. RBONE-Revista Brasileira De Obesidade, Nutrição E Emagrecimento, 12(71), 391-399, 2018.

22. CAMBI MPC, MARCHESINI SD, BARETTA GAP. Reganho de peso após cirurgia bariátrica: avaliação do perfil nutricional dos pacientes candidatos ao procedimento de plasma endoscópico de argônio. ABCD. Arquivos Brasileiros de Cirurgia Digestiva (São Paulo), v. 28, n. 1, p. 40-43, 2015.

23. Nikiforova I, Barnea R, Azulai S, Susmallian S. Analysis of the Association between Eating Behaviors and Weight Loss after Laparoscopic Sleeve Gastrectomy. Obesity facts, v. 12, n. 6, p. 618-631, 2019.

24. Ruiz-Tovar J, Boix E, Bonete JM, Martínez R, Zubiaga L, Díez M, et al. Effect of preoperative eating patterns and preoperative weight loss on the short-and mid-term weight loss results of sleeve gastrectomy. Cirugía Española (English Edition), v. 93, n. 4, p. 241-247, 2015.

25. Barrea L, Di Somma C, Macchia PE, Falco A, Savanelli MC, Orio F, et al. Influence of nutrition on somatotropic axis: Milk consumption in adult individuals with moderate- 
severe obesity. Clinical Nutrition, v. 36, n. 1, p. 293-301, 2015. Disponível em: https://www.sciencedirect.com/science/article/pii/S0261561415003453. Acesso em: 20 Mar. 2020.

26. RAUTIAINEN S, WANG L, LEE IM, MANSON JE, BURING JE, SESSO HD. Dairy consumption in association with weight change and risk of becoming overweight or obese in middle-aged and older women: a prospective cohort study. The American Journal of Clinical Nutrition, v. 103, n. 4, p. 979-988, 2016. Disponível em: https://academic.oup.com/ajen/article/103/4/979/4662886. Acesso em: 20 Mar. 2020.

27. HANSEN TT, JAKOBSEN TA, NIELSEN MS, SJÖDIN A, LE ROUX CW, SCHMIDT JB. Hedonic changes in food choices following Roux-en-Y gastric bypass. Obesity surgery, v. 26, n. 8, 2016. Disponível em: https://link.springer.com/article/10.1007/s11695-016-2217-x. Acesso em: 20 Mar. 2020.

28. JUSTINO YAC, Tatagiba TNB, Pogian LP, Pimentel FC. Modificações comportamentais entre o pré e o pós-operatório de pacientes bariátricos. Psicol. rev. (Belo Horizonte), Belo Horizonte, v. 24, n. 2, p. 577-599, ago. 2018 . Disponível em http://pepsic.bvsalud.org/scielo.php?script=sci_arttext\&pid=S167711682018000200012\&lng=pt\&nrm=iso. Acesso em: 20 Mar. 2020.

29. Nielsen MS, Andersen IN, Belinda L, Ritz C, le Roux CW, Schmidt JB, et al. Bariatric Surgery Leads to Short-Term Effects on Sweet Taste Sensitivity and Hedonic Evaluation of Fatty Food Stimuli. Obesity (Silver Spring) ; v. 27, n. 11, p. 1796-1804, 2019. Disponível em: https://pesquisa.bvsalud.org/portal/resource/pt/mdl-31556242. Acesso em: 20 Mar. 2020.

30. Kittrell H, Graber W, Mariani E, Czaja K, Hajnal A, Di Lorenzo PM. Taste and odor preferences following Roux-en-Y surgery in humans. PLoS One. v. 13, n. 7, 2018. Disponível em: https://pubmed.ncbi.nlm.nih.gov/29975712/. Acesso em: 20 Mar. 2020.

31. YANOS BR, SAULES KK, SCHUH LM, SOGG S. Predictors of lowest weight and long-term weight regain among Roux-en-Y gastric bypass patients. Obesity surgery, v. 25, n. 8, p. 1364-1370, 2015. Disponível em: https://link.springer.com/article/10.1007\%2Fs11695-014-1536-z. Acesso em: 20 Mar. 2020.

32. Hubert PA, Papasavas P, Stone A, Swede H, Huedo-Medina TB, Tishler D, et al. Associations between Weight Loss, Food Likes, Dietary Behaviors, and Chemosensory Function in Bariatric Surgery: A Case-Control Analysis in Women. Nutrients, v. 11, n. 4, 2019. Disponível em: https://pubmed.ncbi.nlm.nih.gov/30970617/. Acesso em: 20 Mar. 2020.

33. Shoar S, Naderan M, Shoar N, Modukuru VR, Mahmoodzadeh H. Alteration Pattern of Taste Perception After Bariatric Surgery: a Systematic Review of Four Taste Domains. Obes Surg. v. 29, n. 5, p. 1542-1550, 2019. Disponível em: https://pubmed.ncbi.nlm.nih.gov/30712168/. Acesso em: 20 Mar. 2020.

34. Ahmed K, Penney N, Darzi A, Purkayastha, S. Taste Changes after Bariatric Surgery: a Systematic Review. Obes Surg. v. 28, n. 10, p. 3321-3332, 2018. Disponível em: https://pubmed.ncbi.nlm.nih.gov/30062466/. Acesso em: 20 Mar. 2020.

35. Behary P, Miras AD. Food preferences and underlying mechanisms after bariatric surgery. Proc Nutr Soc, v. 74, n. 4, p. 419-425, 2015. Disponível em: https://pubmed.ncbi.nlm.nih.gov/25990312/. Acesso em: 20 Mar. 2020.

36. Tsouristakis AI, Febres G, McMahon DJ, Tchang B, Conwell IM, Tsang AJ, et al. LongTerm Modulation of Appetitive Hormones and Sweet Cravings After Adjustable Gastric Banding and Roux-en-Y Gastric Bypass. Obes Surg. v. 29, n. 11, p. 3698-3705, 2019. Disponível em: https://pubmed.ncbi.nlm.nih.gov/31376135/. Acesso em: 20 Mar. 2020. 
37. Kapoor N, Al-Najim W, le Roux CW, Docherty NG. Shifts in Food Preferences After Bariatric Surgery: Observational Reports and Proposed Mechanisms. Curr Obes Rep., v. 6, n. 3, p. 246-252, 2017. Disponível em: https://pubmed.ncbi.nlm.nih.gov/28699145/. Acesso em: 20 Mar. 2020.

38. Zoon HFA, de Bruijn SEM, Smeets PAM, de Graaf C, Janssen IMC, Schijns W, et al. Altered neural responsivity to food cues in relation to food preferences, but not appetiterelated hormone concentrations after RYGB-surgery. Behavioural Brain Research, v. 353, p. 194-202, 2018. Disponível em:

https://www.sciencedirect.com/science/article/pii/S0166432818306016. Acesso em: 20 Mar. 2020.

39. Han W, Tellez LA, Niu J, Medina S, Ferreira TL, Zhang X, et al. Striatal Dopamine Links Gastrointestinal Rerouting to Altered Sweet Appetite. Cell metabolism, v. 23, n. 1, p. 103-112, 2016. Disponível em: https://pubmed.ncbi.nlm.nih.gov/26698915/. Acesso em: 20 Mar. 2020.

40. Nance K, Acevedo MB, Pepino MY. Changes in taste function and ingestive behavior following bariatric surgery. Appetite, v. 146, 2020. Disponível em: https://pubmed.ncbi.nlm.nih.gov/31473274/. Acesso em: 20 Mar. 2020.

41. Van Vuuren MAJ, Strodl E, White KM, Lockie PD. Taste, Enjoyment, and Desire of Flavors Change After Sleeve Gastrectomy-Short Term Results. Obesity surgery, v. 27, n. 6, p. 1466-1473, 2017. Disponível em: https://pubmed.ncbi.nlm.nih.gov/27981459/. Acesso em: 20 Mar. 2020.

42. El Labban S, Safadi B, Olabi A. The Effect of Roux-en-Y Gastric Bypass and Sleeve Gastrectomy Surgery on Dietary Intake, Food Preferences, and Gastrointestinal Symptoms in Post-Surgical Morbidly Obese Lebanese Subjects: A Cross-Sectional Pilot Study. Obesity surgery, v. 25, n.12, p. 2393-2399, 2015. Disponível em: https://pubmed.ncbi.nlm.nih.gov/25982803/. Acesso em: 20 Mar. 2020.

43. ZARSHENAS N, TAPSELL LC, NEALE E, BATTERHAM M, TALBOT ML. The Relationship Between Bariatric Surgery and Diet Quality: a Systematic Review. Obesity Surgery. 2020. Disponível em: https://pubmed.ncbi.nlm.nih.gov/31940138/. Acesso em: 20 Mar. 2020.

44. Smith KR, Papantoni A, Veldhuizen MG, Kamath V, Harris C, Moran TH, et al.. Tasterelated reward is associated with weight loss following bariatric surgery. The Journal of Clinical Investigation. v. 1, 2020. Disponível em: https://www.jci.org/articles/view/137772. Acesso em: 20 Mar. 2020.

45. Nance K, Acevedo MB, Pepino MY. Changes in taste function and ingestive behavior following bariatric surgery. Appetite, v. 146, 2020. Disponível em: https://pubmed.ncbi.nlm.nih.gov/31473274/. Acesso em: 20 Mar. 2020.

46. Goldstone AP, Miras AD, Scholtz S, Jackson S, Neff KJ, Pénicaud L, et al. Link Between Increased Satiety Gut Hormones and Reduced Food Reward After Gastric Bypass Surgery for Obesity. The Journal of Clinical Endocrinology \& Metabolism, v. 101, n. 2, p. 599-609, 2016 Disponível em: https://academic.oup.com/jcem/article/101/2/599/2810906. Acesso em: 20 Mar. 2020.

47. Nielsen MS, Andersen INSK, Lange B, Ritz C, le Roux CW, Schmidt JB, et al. Roux-EnY Gastric Bypass and Sleeve Gastrectomy Does Not Affect Food Preferences When Assessed by an Ad libitum Buffet Meal. Obesity surgery, v. 27, n. 10, p. 2599-2605, 2017. Disponível em: https://pesquisa.bvsalud.org/portal/resource/pt/mdl-31556242. Acesso em: 20 Mar. 2020.

48. Sudan R, Sudan R, Lyden E, Thompson JS. Food cravings and food consumption after Roux-en-Y gastric bypass versus cholecystectomy. Surgery for obesity and related diseases : official journal of the American Society for Bariatric Surgery, v. 13, n. 2, p. 
220-226, 2017. Disponível em: https://pubmed.ncbi.nlm.nih.gov/27771313/. Acesso em: 20 Mar. 2020.

49. El Labban S, Safadi B, Olabi A. Effect of Roux-en-Y gastric bypass and sleeve gastrectomy on taste acuity and sweetness acceptability in postsurgical subjects. Nutrition. v. 32, p.1299-1302, 2016. Disponível em:

https://pubmed.ncbi.nlm.nih.gov/27264159/. Acesso em: 20 Mar. 2020.

\section{Como citar este artigo:}

Dias LF, Detoni ACT, Andrade GLC, Alves CV, Campos JVSM, Araújo JMM. Efeitos da Cirurgia Bariátrica no Consumo de Doces e Alimentos Gordurosos por Pacientes em seu Período Pré e Pós-Operatório. Rev. Aten. Saúde. 2021; 19(69): 195-212. 